\title{
Effects of temporal dynamics on perceived authenticity of smiles
}

\author{
David Horic-Asselin $^{1} \cdot$ Patricia Brosseau-Liard ${ }^{1} \cdot$ Pierre Gosselin $^{1} \cdot$ Charles A. Collin $^{1}$
}

Published online: 28 June 2020

(C) The Psychonomic Society, Inc. 2020

\begin{abstract}
We presented participants with videos of Duchenne smiles that differed in the duration of their onset, offset, or both to determine if this would affect perceived expression authenticity. The duration of onset and offset varied between 0.2 and $1.0 \mathrm{~s}$. Participants were shown one smile at a time and were asked to judge its genuineness on a rating scale. Results indicated the duration of offset had an effect on perceived genuineness when it was manipulated in isolation. Similarly, when both the offset and onset duration were adjusted concomitantly, genuineness ratings were affected. There was no effect of onset duration when it was manipulated in isolation. This is the first demonstration of these effects using photographs of real human faces that are dynamically and morphologically symmetrical, and which have been validated via the Facial Action Coding System.
\end{abstract}

Keywords Temporal dynamics $\cdot$ Authenticity $\cdot$ Genuineness $\cdot$ Smiles $\cdot$ Facial expressions $\cdot$ Perception of emotions

\section{Introduction}

Emotions are not always transparently expressed through facial activity. Although many studies do support the claim that there is a concordance between the felt emotion and the expressed one (Gosselin et al., 1995; Rosenberg \& Ekman, 1994; Ruch, 1995), facial activity can also be controlled voluntarily to display non-genuine emotional expressions. It has been suggested that there was a time in human evolution where there was a perfect concordance between emotions and facial expressions (Owren \& Bachorowski, 2001), but that since then Hominidae have acquired the ability to produce facial expressions of emotions voluntarily. Among the numerous emotional expressions that can be voluntarily produced, the smile is arguably the most ubiquitous and has an important role to play in social interactions among individuals (Bugental, 1986; Niedenthal et al., 2010).

According to Owren and Bachorowski (2001), smiling reinforces positive feelings towards other individuals, which increases the chances of receiving a favorable treatment in return. This positive feedback is recursive and, over time, reinforces attachment between individuals. It is thought that early humans eventually developed the ability to use false

David Horic-Asselin

David.asselin@uottawa.ca

1 School of Psychology, University of Ottawa, 136 Jean-Jacques Lussier Vanier Hall, Ottawa, Ontario K1N 6N5, Canada smiles in order to get resources from others. The ability to distinguish between false and genuine smiles then appeared and evolved over time. It is, however, only in the last 40 years or so that this distinction between genuine and non-genuine smiles has elicited serious interest from researchers.

In the context of this article, we use the term "genuine smile" to designate the case where there is a concordance between what an individual feels and what they show through their facial activity. That is, a genuine smile occurs when an individual is happy. On the other hand, we use the term "false smile" to describe when a person smiles in order to make others believe they are happy even though they are not. The terms "authentic smile," "genuine smile," and "Duchenne smile" are used interchangeably. These terms are similar, but not identical, to the terms "emitted expression" versus "elicited expression" used by other researchers (McGettigan et al., 2015). Studies conducted since the early 1980 s have found many differences between genuine and false smiles, including differences in the action units that are activated during a facial expression, their symmetry, and their temporal dynamics (Ambadar et al., 2005; Ekman et al., 1980, 1988, 1990; Ekman \& Friesen, 1974; Wehrle et al., 2000). The latter is of primary concern in the present article.

Studies of facial expressions of emotion have primarily used static images portraying the culmination of the emotional display as stimuli. While this has been useful as a means to detect differences in behaviour and performance elicited by the various emotional expressions, it is not particularly useful for the judgment of authenticity because this is not how emotional displays are reflected in daily life. That is, such 
maximum-intensity static images of facial expressions lack ecological validity. For that reason, in the current study we opted to examine the effects of temporal dynamics on the interpretation of judgments of genuineness of smiles. Below, we discuss previous findings regarding differences between authentic and false smiles, culminating in a review of the literature on differences in temporal dynamics. As is shown, the results in this part of the literature are mixed, possibly because of differences in stimulus qualities. We aimed to address this by using video stimuli of real human facial expressions of happiness that are validated through the Facial Action Coding System (FACS; Ekman \& Friesen, 1982; Ekman et al., 2002), as well as being dynamically and temporally symmetrical. We reasoned that doing so would yield results that can be generalized to the interpretation of smile genuineness in everyday life.

\section{Differences between authentic and false smiles}

Action units Most studies that have tried to identify the differences between genuine and false smiles elicited an emotional reaction from participants using videos, interviews, or hypnosis and filmed their reactions in order to codify them with the Facial Action Coding System (Ekman et al., 1980, 1988, 1990; Ekman \& Friesen, 1974; Krumhuber \& Manstead, 2009; Weiss et al., 1987). The FACS is a tool that was developed to codify the different muscle groups in the face, called action units $(A U s)$, and contains norms about the intensity, symmetry, and temporal dynamics of their activations. With the exception of Krumhuber and Manstead (2009), all previous studies reported the activation of the Cheek Raiser and Lip Corner Puller action units during an authentic smile. Another study (Gosselin et al., 1995) examined the occurrence of the Cheek Raiser and the Lip Corner Puller action units when actors, using the Stanislavski method, produced smiles while feeling happiness. Significantly more AUs associated with the authentic smile were activated when the happiness was felt (93\%) compared to when it was simulated (84\%). In order to distinguish false from authentic smiles, Ekman and Friesen (1982) suggested a few facial markers. The most wellknown and well-studied is the Duchenne marker, which involves the activation of the Cheek Raiser AU. This has been considered a very reliable marker to distinguish authentic from false smiles, though there is still much debate regarding the use of this $\mathrm{AU}$ as the barometer for authentic smiles. For instance, several researchers have observed that, on average, between $17 \%$ and $30 \%$ of individuals can voluntarily control this muscle activation (Gosselin et al., 2002; Gunnery et al., 2013; Krumhuber et al., 2009; Schmidt and Cohn, 2001a, b; Schmidt et al., 2006b). Even though a small proportion of people can voluntarily produce the Duchenne marker, a large majority of authentic smiles are still composed of both the lip corner puller and the cheek raiser AUs, and these are therefore assumed to be valid markers of expression genuineness (Ekman, 1993).

Symmetry The second cue that researchers have studied to distinguish authentic from false smiles is the symmetry of the facial expression. Symmetry here refers not to the static morphological differences between the left and the right side of an individual's face, but rather to the difference in symmetry produced between the left and right sides during a dynamic facial expression of emotion (Borod et al., 1988; Borod \& Caron, 1980; Brockmeier \& Ulrich, 1993; Ekman et al., 1981; Hager \& Ekman, 1985, 1997; Okamoto et al., 2010; Schmidt et al., 2006a, 2009, 2009; Sirota \& Schwartz, 1982). The majority of these studies confirmed Ekman's (1980) hypothesis, which suggests that an authentic smile tends to be produced in a symmetrical fashion, while a false smile involves more asymmetrical AU activations.

Temporal dynamics Dynamic information is thought to be important in everyday decoding of emotional expressions and assessments of their authenticity. Several studies have addressed the role of dynamic information in the interpretation of facial expressions, but only a few have specifically addressed its effect on judgments of authenticity. Indeed, just a handful of studies (Bruce \& Valentine, 1988; Kamachi et al., 2013; Wehrle et al., 2000) have demonstrated that dynamic characteristics provide useful information for the judgment of genuine smiles. One reason that such studies have been relatively rare is that creating well-controlled dynamic stimuli has, until recently, been quite difficult. Modern advances in technology have shown promise to diminish the problems associated with creating such stimuli, but it is still today a costly endeavor. One of the first studies examining the information provided by the dynamic properties of facial stimuli was conducted by Bassili (1978). His results showed that observers were better at identifying a face when a moving configuration of lights was used compared to a static configuration. More recently, Lander and Bruce (2004) showed that the recognition of faces is better when their temporal dynamic remains unmodified, and that changing the rhythm of presentation reduced the recognition of faces.

Regarding facial expression, several studies have shown that dynamic properties differ between genuine and false smiles (Bruce \& Valentine, 1988; Cohn \& Schmidt, 2004; Frank et al., 1993; Hess et al., 1989; Hess \& Kleck, 1990; Kamachi et al., 2013; Krumhuber \& Kappas, 2005; Schmidt et al., 2006b, 2009; Wehrle et al., 2000). However, this alone does not show that humans are capable of detecting such differences or of using them to assess genuineness. To decode such differences, we first need to detect and interpret these cues. A few studies have demonstrated that the dynamic cues might not always be perceived or correctly interpreted by individuals (Hess et al., 1989; Hess \& Kleck, 1994). For 
example, Hess and Kleck (1990) found that the duration of onset or offset did not have any effect on the accuracy of smile discrimination as their data showed no main effects or interactions for average onset and offset time. It is also possible that the wrong cues are used when attempting to distinguish between false and genuine smiles. Hess and Kleck (1994) found that that their participants were only somewhat better at identifying spontaneous expressions from posed expressions. While they were able to accurately identify spontaneous expressions of happiness and disgust at above-chance level, their performance significantly dropped for posed expressions of the same emotions, which they suggest to be the result of using invalid cues, like facial morphology or hairstyles (vs. AU activation patterns, for example). However, in the results of this study the evaluated cues were collapsed across two emotions, happiness and disgust, making it impossible to know how much the results apply to smiles in particular.

Total duration The duration of a smile, including all three of its different phases (onset, apex, offset), can simply be measured using recorded videos and quantified in seconds or milliseconds. The majority of researchers who have studied the temporal dynamics of smiles have used this approach (Bugental, 1986; Cohn \& Schmidt, 2004; Frank et al., 1993; Hess et al., 1989; Hess \& Kleck, 1990; Krumhuber \& Kappas, 2005; Schmidt et al., 2006b, 2009; Weiss et al., 1987). Ekman $(1980,1982)$ first proposed that the mean duration of an authentic smile was around $4 \mathrm{~s}$ and that it rarely is shorter than $0.33 \mathrm{~s}$. He added that the duration of the authentic smile is correlated with the degree of happiness. That is, a less authentic smile would tend to be shorter, while a more authentic smile would be longer, and nearer $4 \mathrm{~s}$ duration. Indeed, studies confirm this (Cohn \& Schmidt, 2004; Ekman et al., 1980; Frank et al., 1993; Mavadati et al., 2016; Schmidt and Cohn, $2001 \mathrm{a}, \mathrm{b}$ ); the mean duration of authentic smiles varies on average between 0.66 and 4.75 s. Conversely, past studies have shown evidence that false smiles last longer than authentic ones, with a mean duration of $7.09 \mathrm{~s}$ (Frank et al., 1993; Hess \& Kleck, 1990).

Onset, apex, and offset While the smile can be taken as a whole, it is also possible to analyze it on a finer scale and discriminate three different phases within it. The onset duration refers to the length of time from the start of the smile until its maximum intensity, the apex duration is the length of time for which the intensity remains at its maximum, and the offset duration refers to the length of time from the end of the apex until the smile ends. Past literature suggests that the onset duration of a false smile is shorter than that of an authentic one. The mean duration for authentic smile onset varies between $0.49 \mathrm{~s}$ and 0.93 s (Bugental, 1986.; Cohn \& Schmidt, 2004; Mavadati et al., 2016; Schmidt et al., 2003, 2006a, 2009; Tarantili et al., 2005), whereas the mean onset duration of a false smile varies from 0.54 s to 0.77 s (Bugental, 1986; Cohn \& Schmidt, 2004; Schmidt et al., 2006b, 2009; Weiss et al., 1987).

With regard to the effects of the duration of the offset of smiles on their perceived authenticity, there does not seem to be a general consensus in the literature and we believe more work needs to address this with more valid and consistent stimuli. It was found by Bugental (1986) that the offset duration of authentic smiles $(2.39 \mathrm{~s})$ was longer than that of false smiles $(1.63 \mathrm{~s})$, but opposite results were found by Schmidt, Ambadar, et al. (2006b), with findings of 0.56-s and 0.64-s durations for authentic and false smiles, respectively. This discrepancy is most likely due to methodological differences. For instance, Bugental (1986) used a complex method for eliciting genuine and false smiles. Contrary to most recent research in this field, she did not control the social context in which the smiles were produced. Instead, she elicited interactions between elementary school-aged boys and adults by training some of the boys to be responsive to the adults, while other boys were chosen based on their unresponsive dispositions.

A similar study to ours (Krumhuber \& Kappas, 2005) used Poser, a computer graphics program used to create artificial images of human faces, to examine the effect of onset, apex, and offset duration on ratings of smile genuineness. Using the same duration parameters from two previous studies (Cohn \& Schmidt, 2004; Schmidt and Cohn, 2001a, b), they evaluated a segment of the smile specifically or a combination of segments, and found the temporal dynamic to influence the judgment of authenticity. More precisely, smiles with longer onsets and offsets were rated as more genuine, and the authenticity decreased as the duration augmented past a certain point. A limitation of this particular study was the use of computergenerated male-only models; past literature shows evidence of gender stereotype effects in the judgment of facial expression, and particularly the smile (Hess et al., 2000, 2005). Also, while computer-generated 3D models of facial expressions are getting better at mimicking the real human face, they are still not as easily recognized as photographs of real human faces (Chamberland \& Collin, 2020). Krumhuber et al.' (2012) results showed that artificial emotional facial expressions were rated by subjects to be about $70 \%$ comparable to their real human equivalent, and about $80 \%$ when it comes to the neutral facial expression (Amini \& Lisetti, 2013; Krumhuber et al., 2012). This suggests that they would elicit poorer performance than photographs in terms of emotional expression categorization, although no previous study has examined this specifically.

Indeed, there is currently no evidence that computergenerated facial expressions are comparable to photographs of real human expressions in terms of recognition rates. Furthermore, as discussed in Roesch et al. (2011), even if individuals are able to accurately predict the emotional label associated with a computer-generated facial expression, and even if these are FACS validated, this is not enough to validate 
the use of such stimuli for more complex interpretation tasks like judgments of authenticity (Roesch et al., 2011). Finally, in a recent study, Chamberland and Collin (2020) compared photos from the Standard Expressor Version JACFEE (SEV-JACFEE) to FaceGen Modeller Core v3.18 (Singular Inversions, Toronto, ON, Canada) and found a significant difference in both accuracy of expression categorization and subjective ratings of emotion intensity between computergenerated facial expressions and photos. This was the case for most expressions, including happiness. Their results also suggested that there was confusion in terms of the information provided by the eye region of the face; this would be a serious impediment to the use of computer-generated facial expressions in studies on smile genuineness, because the eye region is thought to show the marker that distinguishes false from authentic smiles in real human facial expressions. Altogether, the previous results support the affirmation that humans face stimuli have a better ecological validity and should be preferred to using computer-generated ones.

Finally, as for the apex segment of the smile, it has hardly been studied at all. The one study we are aware of that has examined this aspect of the smile duration (Hess \& Kleck, 1990) found no significant differences in apex duration between false and genuine smiles. This is contrary to Ekman and Friesen's (1982) hypothesis suggesting that the apex duration of an authentic smile should be shorter than the corresponding version of a false smile. In respect to the duration of the onset and offset segments of the smile, to our knowledge, no other studies have attempted to manipulate both simultaneously.

In summary, previous work shows mixed results regarding the effects of the duration of different phases of smiles on their perceived authenticity. However, considering the literature as a whole, the results suggest that the onset duration and the offset duration of an authentic smile should both be generally longer than those of a false smile.

\section{Objectives}

The main objective of this study was to investigate whether the duration of a genuine smile has an effect on its perceived authenticity. More specifically, we wanted to determine if manipulating the durations of the individual temporal segments of a genuine smile (onset and offset) would influence judgments of its authenticity in different ways. The present study differs methodologically from previous studies (Krumhuber et al., 2007; Krumhuber \& Kappas, 2005; Sato \& Yoshikawa, 2004, 2009) in that we created stimuli of both genders that were photographic in nature and well validated according to the FACS. Moreover, our stimuli were both spatially and temporally symmetrical, in that AU activations were equal across face halves and smile onset and offset timings symmetrical. We decided to use all Duchenne smiles to control its possible effect as some studies have suggested that the
Duchenne markers could be a marker of smile intensity rather than smile authenticity (Girard, Shandar, Liu, Cohn, Yin, and Morency, 2019; Krumhuber \& Manstead, 2009). Since symmetrical smiles are usually perceived as being more authentic, we wanted to apply the symmetry to both the AUs used and the duration of the phases of the smiles, which makes our stimuli completely symmetrical both spatially and temporally (the axis being the peak of the smile). While the spatial symmetry of smiles has been investigated quite extensively, no studies we are aware of examine their temporal symmetry. We also decided to create stimuli that were void of any neutral facial expression segments. That is, our stimuli start as soon as there is movement within the face, and ends immediately when the movement stops. It can be debated that a neutral facial display is only very rarely, if ever, seen in our daily lives and could potentially interfere with the participants' interpretation of the emotional facial expressions, especially when included in an authenticity judgment task.

As the literature is fairly consistent concerning the onset (a longer onset is judged as more authentic), and because there is no clear consensus regarding the offset, we decided to use the same parameters for both phases of the smile to see if we observe a similar effect. We chose to manipulate onset and offset durations to values of $0.2,0.4,0.6,0.8$, and $1.0 \mathrm{~s}$, because this span encompasses the values found by the most recent literature for genuine and false smiles (Schmidt et al., 2006a, 2006b, 2009). Although findings in the literature regarding the durations of the various phases of genuine and false smiles are inconsistent, we predicted that longer onset and offset durations of our genuine smile stimuli would be associated with a higher perceived genuineness rating. This is based on the idea that genuine smiles with short onset/offset durations would provide a lower signal strength. They could therefore be judged as being more ambiguous and potentially less authentic. A second objective of this study was to investigate the perceptive abilities of individuals with regard to discriminating different durations of smiles and their temporal segments. That is, we also wanted to identify the minimum duration difference that leads to a difference in perceived smile authenticity.

For the purposes of this article, a genuine smile is defined as one that contains the following validated characteristics: (1) Action units 6 (Cheek Raiser - orbicularis oculi) and 12 (Lip Corner Puller - Zygomatic Major) are activated, (2) they are activated symmetrically, and (3) they are activated within the appropriate duration range.

\section{Method}

\section{Participants}

We performed an a priori power analysis using GPower (Faul et al., 2007) to estimate our sample size for a repeated- 
measures ANOVA (five levels, power $=0.95, \alpha=0.05$ ). This indicated that 30 participants would be sufficient. The participants were 40 adults ( 27 females, 13 males) ranging in age from 17 to 27 years $(M=19.5, S D=1.61)$. All were recruited from the participant pool of a public university and received course credit for participating in the study. The participants were all French speaking.

\section{Stimulus materials}

Three models were recruited in order to create the stimuli for this research. A Facial Action Coding system (FACS; Ekman \& Friesen, 1982, Ekman et al., 2002) expert (the third author of this manuscript, $\mathrm{PG}$ ) who underwent extensive training and completed a FACS proficiency test, met individually with each model and gave him or her proper instructions for activating specific facial action units. Thus, the physical parameters of the smiles were controlled with the FACS. The FACS contains norms to code the intensity of action units and their temporal dynamics. Five intensity levels, from A (very slight) to $\mathrm{E}$ (extreme), and three temporal phases (onset, apex and offset) were distinguished. Each smile was described in words by the FACS expert and illustrated with photographs. Then, the models were instructed to practice the target smiles while looking at their face in a mirror and receiving feedback from the FACS expert. After a few successful attempts, the models were videotaped while producing each of the three smiles 30 times, receiving feedback after each attempt.

The videos were subsequently coded by three coders who had previously passed the FACS proficiency test. The genuine smile involved the activation of Cheek Raiser and Lip Corner Puller. The former raises the cheeks, causing crows' feet and bulges beneath the eyes, while the latter pulls lip corners up diagonally towards the cheekbones. The models were instructed to activate moderately (C level) and symmetrically these two AUs for each side of the face, with onset and offset lasting about $1 \mathrm{~s}$. As discussed above, a smile corresponding to those parameters is also known as the Duchenne smile ( Ekman, 1993; Ekman \& Friesen, 1982; Frank et al., 1993).

Selection and construction of the stimuli From among the smiles that were approved by the FACS coders, three were selected to be used for the current study. One of the models was female and two were male. The smiles were then manipulated using a video-editing program to alter the durations of the different temporal segments (onset, offset, or both) to correspond to five distinct values: $0.2,0.4,0.6,0.8$, and $1.0 \mathrm{~s}$. To maintain the realism of the original Duchenne smiles while changing presentation durations, the video clips were deconstructed into individual frames, and frames at regular intervals were removed before reconstructing the remaining ones into a video clip that matched the temporal requirements of each experimental condition. For instance, to create the .2 -s onset version of a smile, every fifth frame was kept from the original 1-s onset and all others were discarded. There were some minor variations from this regularity that were imposed by the fact that the original onset and offset were not exactly $1 \mathrm{~s}$ in duration. The final stimuli all had the same duration $( \pm$ $0.001 \mathrm{~s}$ ). See Fig. 1 for an example of the final frame of a stimulus onset.

\section{Procedure}

After participants arrived at the laboratory, they were seated at a desk with a keyboard, mouse, and a 23 -in. computer screen, placed $60 \mathrm{~cm}$ away from them. The experiment was automated using Superlab Pro 5 (Cedrus), a stimulus presentation application. The difference between a genuine and a fake smile was explained using two contextual situations. It was explained to participants that a person can feel happy and openly show it, in which case the smile would be authentic. In a different context, a person can smile yet not feel happy because he does not want others to know he is not happy. In this case, the smile would be fake. They were then told they would see smiles and they would need to rate their authenticity on a scale. It was indicated that there were no right or wrong answers.

This experiment was divided into three parts (onset, offset, and onset+offset). In the onset condition, only the onset duration was manipulated, and the participants were asked to pay attention to the moment between the start of the smile, its neutral state, and the moment when it was most intense. The onset duration was one of five values: $200,400,600,800$, or $1,000 \mathrm{~ms}$. The apex and the offset durations remained fixed at $1,500 \mathrm{~ms}$ and $1,000 \mathrm{~ms}$, respectively. In the offset condition, only the offset duration was manipulated, again such that it lasted 200, 400, 600, 800, or 1,000 ms. Participants were asked to pay attention to the moment between when the smile

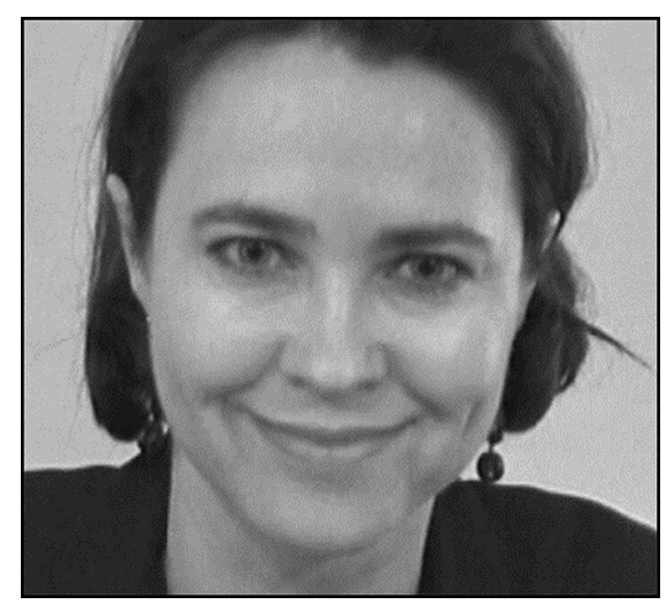

Fig. 1 Example of a frame from stimulus videos. This is the final frame of the smile onset, also known as the apex of a smile, when its intensity is at its highest 
was most intense and the end of the smile, when it returned to a neutral state. The apex and the onset durations remained fixed at 1,500 $\mathrm{ms}$ and 1,000 ms, respectively. Finally, in the offset+onset condition, participants were asked to pay attention to the whole smile. Both onset and offset were simultaneously manipulated to both last $200,400,600,800$, or $1,000 \mathrm{~ms}$ while the apex duration remained fixed at $1,500 \mathrm{~ms}$.

Following the presentation of each stimulus, participants were asked to rate the genuineness of the smile by clicking on the appropriate button on a five-point scale ranging from least to most authentic. The order of presentation for the three conditions was counterbalanced and the order of trials within each condition was randomized. Every model $(3) \times$ duration (5) was presented three times in each of the three conditions. Thus, the entire experiment consisted of 135 trials. The experimental sessions were done individually and the responses for each participant were recorded automatically.

\section{Results}

The mean rating of authenticity was calculated for each duration and each condition (onset, offset, both). Because all assumptions of analysis of variance were met, we proceeded with a one-way repeated-measures ANOVA with five levels to examine the effect of the duration on the perceived genuineness of smiles. Below we discuss findings for onset, offset, and onset+offset conditions in that order.

\section{Onset duration}

In analyzing data from the onset condition, we found no evidence that smiles with shorter onset were perceived as less authentic than those with longer ones. This finding was unexpected, as one might predict that individuals would use the first moments of a smile to judge whether it is authentic or not. Instead, our data, as seen in Fig. 2, showed no evidence that individuals are sensitive to a change in duration of the onset, $F(4,156)=2.241, p=.105$, even when it is one-fifth of its normal span.

\section{Offset duration}

In contrast to onset duration data, we found a clear and significant main effect of offset duration $F(4,156)=23.23, p<.001$ (see Fig. 3). In agreement with Ekman (2003), Holm-Sidak's multiple comparison tests indicated that smiles with offsets of $1 \mathrm{~s}$ were rated as more genuine than those with offsets of $.2(p$ $<0.001), .4(p<0.001)$, and $.6 \mathrm{~s}(p=0.007)$. The smiles with an offset of $.2 \mathrm{~s}$ were judged to be less authentic than their longer versions. The mean rating of the latter was 2.30 while the average for the 1-s condition was 3.05.

The difference between the offsets had, in general, to be of $.4 \mathrm{~s}$ or more to produce a statistically significant effect on participants' judgment ratings. Exceptionally, the participants were able to distinguish between offsets of $.2 \mathrm{~s}$. and $.4 \mathrm{~s}$. ( $p<$ 0.002 ). However, no other significant differences were found between smiles with an offset contrast of less than $.4 \mathrm{~s}$.

\section{Onset + offset duration}

As with the offset condition, the participants were found to be sensitive to the duration of the smile when the duration of both offset and onset varied, $F(4,156)=7.73, p<.001$, but not as much as in the condition where only the offsets varied (see Fig. 4). Holm-Sidak's multiple comparison tests reveals that smiles with onsets and offsets of .6 $(p=0.013), .8(p=0.014)$,

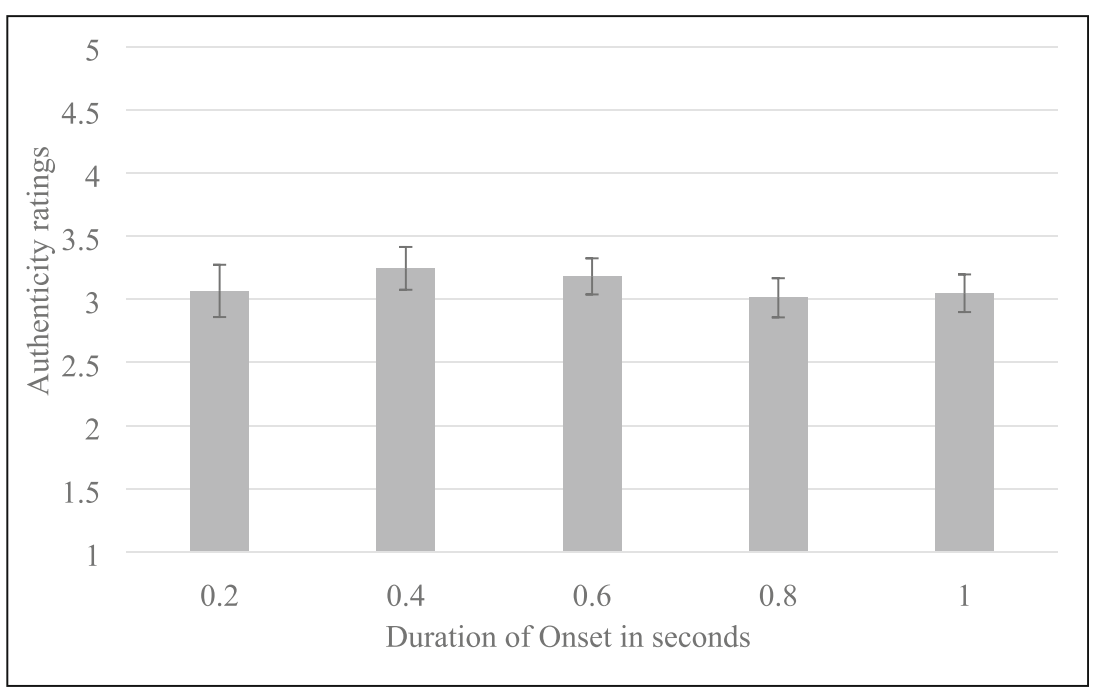

Fig. 2 The effect of onset duration on authenticity ratings 


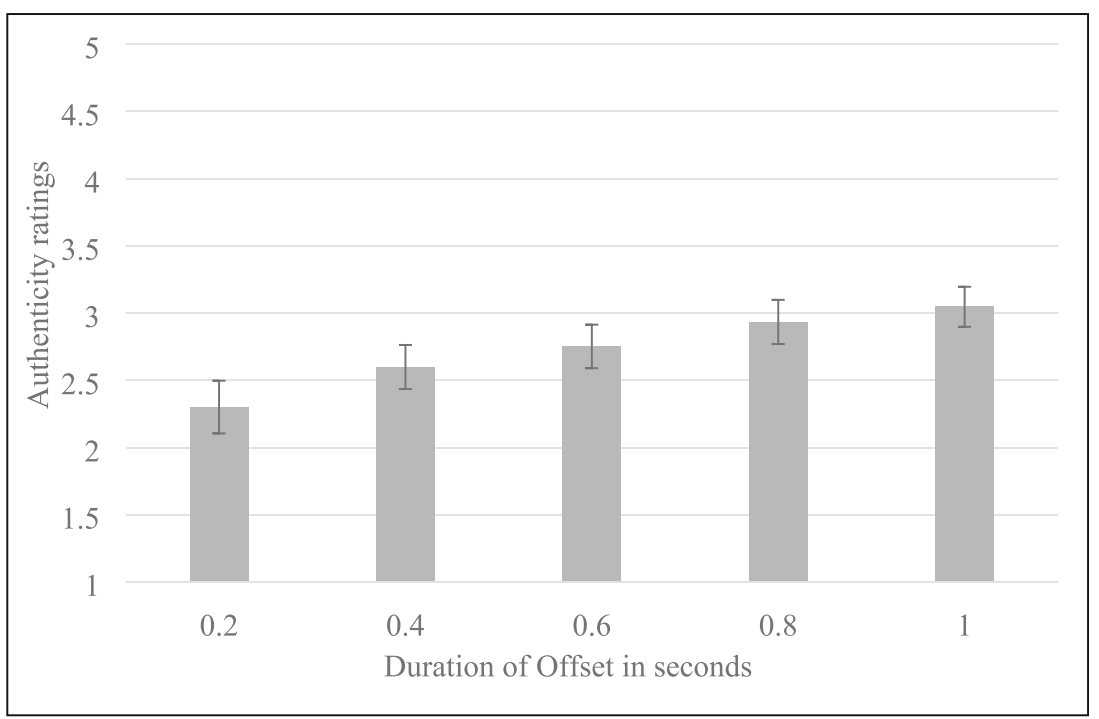

Fig. 3 The effect of Offset duration on authenticity ratings

or $1 \mathrm{~s}(p=0.023)$ were rated as more genuine than those with offsets of $.2 \mathrm{~s}$. No other significant differences were found.

\section{Discussion}

The goal of the present study was to investigate the effects of changes in temporal dynamics on the perceived authenticity of genuine human smiles. In doing so, we wanted to avoid using computer-generated facial expressions as they seem to be of questionable validity relative to photographs of faces (Chamberland \& Collin, 2020; Roesch et al., 2011). We examined whether the duration of the onset and offset of a smile had an effect on judgments of authenticity. The results confirm that manipulations of temporal dynamics can have such an influence.

There was a clear and statistically significant effect of the offset duration as well as the onset+offset duration on the perceived genuineness of our smile stimuli. Surprisingly, manipulations of the onset duration alone had no significant effect on the judgment of authenticity. This is inconsistent with the past literature (Bugental, 1986; Cohn \& Schmidt, 2004; Krumhuber \& Kappas, 2005; Schmidt et al., 2003, 2006a, 2009; Tarantili et al., 2005), which found that authentic smile onsets lasted longer on average than false smile onsets. However, it should be noted that most of the latter studies used artificial facial expressions and models, and that most of their stimuli showed only the isolated segments of the

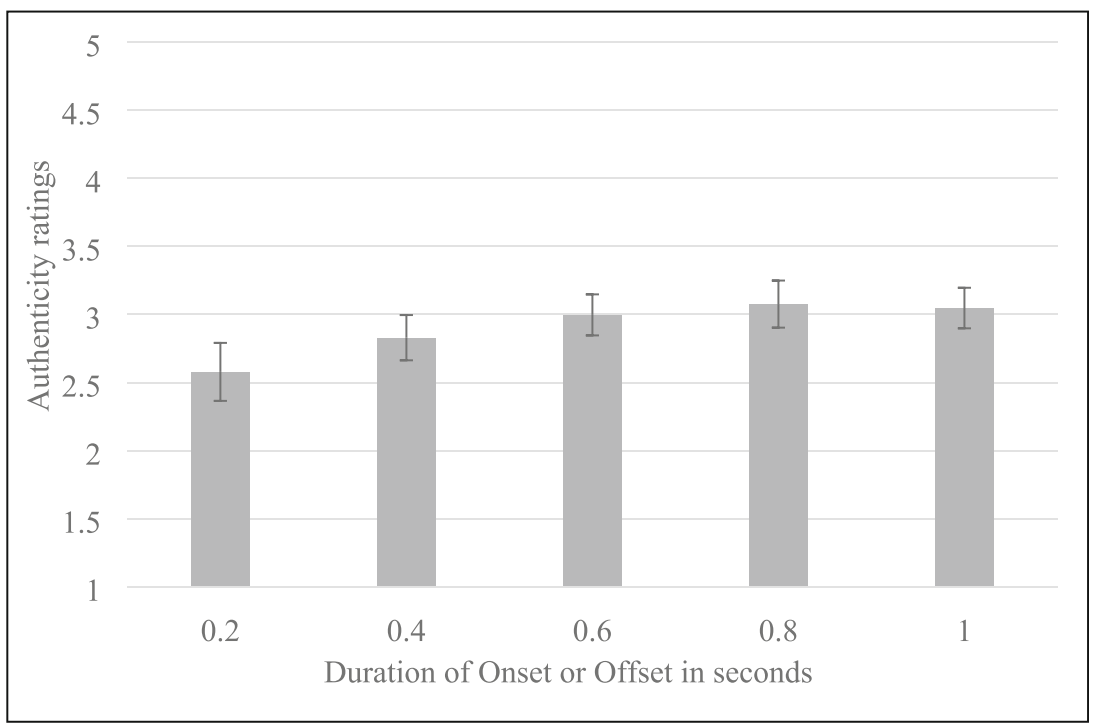

Fig. 4 The effect of Offset duration on authenticity ratings 
smiles (i.e., just the onset or just the offset) and not the whole smile.

While the previous literature does not show any general consensus regarding the effect of the duration of the offset on the perceived authenticity of smiles, we found a very clear pattern whereby a longer offset duration was judged as significantly more authentic than a shorter one. This is in agreement with results obtained from Bugental (1986). Her results suggest that the offset of an authentic smile lasts longer than that of a false smile, though it should be noted that her measured durations were both well above our maximum duration of $1 \mathrm{~s}(2.39 \mathrm{~s}$ and $1.63 \mathrm{~s}$, respectively). Our results contradict those of Schmidt et al. (2006a), which suggested that the offset of an authentic smile is shorter than that of a false smile. This is despite the fact that our tested range contained the values they found ( $0.56 \mathrm{~s}$ and $0.64 \mathrm{~s}$ for genuine and false smiles, respectively).

As for the onset+offset condition, this is the first time to our knowledge that such a manipulation has been examined. Our findings in this condition could be interpreted as a mixture of those found for onset and onset in isolation. That is, we find an effect of duration of onset+offset, but it is somewhat smaller than that found for offset alone. This suggests that manipulating the onset along with the offset in some way reduced the effect of the offset manipulation.

Why is it that the offset duration produced an effect on the judgment of authenticity of smiles but not the onset? Although the present study cannot answer this definitively, several possibilities present themselves. For instance, one plausible explanation is that this is simply a recency effect. The offset might have more of an impact simply because, being the most recent information in memory, it is better recalled. This might be expected to have a particularly pronounced impact in facial emotion processing, where information is complex and ambiguous, and thus may test the limits of cognitive resources. Similarly, it could be that due to cognitive limitations and the large amount of information conveyed in real time by humans' facial activity, we have learned to use only what is most recently available to us (Deese \& Kaufman, 1957; Murdock, 1962). We could also speculate that the beginning of a smile is more often mixed with other emotions due to its occurrence in various social contexts, whereas the end phase tends to contain fewer mixed facial expressions and might be seen as a more reliable marker. A third explanation involves the fact that individuals can control the AUs involved in authentic smiles to a certain extent (Gosselin et al., 2002; Gunnery et al., 2013; Krumhuber et al., 2009; Schmidt and Cohn, 2001a, b; Schmidt et al., 2006b),. It is possible therefore that humans put more emphasis on controlling the beginning of a facial expression, or are just good at doing it for a small amount of time, again for reasons having to do with limits on cognitive resources. For those reasons, individuals may have learned to see the end of a facial expression as more relevant when it comes to judging its genuineness. In summary, it is possible that the offset is simply more informative about the genuineness of a smile, and that people have unconsciously learned to attend to it more when judging authenticity.

Our results are also in line with Ekman's, et al. (1980, Ekman and Friesen, 1982) proposition that the mean total duration of an authentic smile was around $4 \mathrm{~s}$, and that the total duration of the authentic smile is correlated with the degree of happiness (Ekman, Friesen, et al., 1980; Frank et al., 1993). We could certainly refine their affirmations and propose that its ideal duration lies somewhere between $3.5 \mathrm{~s}$ and around $4 \mathrm{~s}$ as our control smile duration is $3.5 \mathrm{~s}$ and is consistently rated as more genuine. Results also indicate that a minimum of $0.4 \mathrm{~s}$ duration difference between smiles was generally needed in order to induce a judgment difference. However, in the case of the offset condition, one exception occurred: The participants gave significantly different genuineness ratings to smiles with a 0.2 -s versus a $0.4-\mathrm{s}$ offset duration. This could once again mean that we are better at gathering important information from the offset than the other segments of the smile. It would be interesting to further validate this finding by testing adults' perceptual abilities, either with a discrimination task between two smiles of different duration or by using a more precise scale. Another follow-up question of interest concerns whether participants would be as good at judging the authenticity of smiles, or at perceptually differentiating the durations of two facial expression stimuli when the models shown are in a real social context versus an experimental setting. We believe our video-editing methodology produced a level of ecological validity that was not reached in past literature. We addressed some of the issues raised by the literature, mainly regarding artificial models and facial expressions. However, as with any experimental study, the models shown to participants were recorded in a laboratory, and were not part of any social context. With recent technological advances, it would be a good idea to use real faces produced in an experimental setting, and implement it artificially in a social context in order to validate even further the necessity of having ecologically valid stimuli, and thus verify if this effect still applies.

Altogether, our results show not only the importance of dynamic properties of smiles but also the temporal aspect or durations of smiles with regard to the judgment of authenticity. These findings support the notion that the dynamic characteristics of a facial expression play an important role in expression interpretation by conveying crucial information about the meaning of an expression to an observer. As only a handful of studies (Bruce \& Valentine, 1988; Kamachi et al., 2013; Krumhuber \& Kappas, 2005; Krumhuber et al., 2009; Wehrle, Kaiser, Schmidt, \& Scherer, 2000) have demonstrated that the dynamic characteristics of the smile provide relevant information for the judgment of its authenticity, this topic of research will need further investigation in order to elucidate the effects of its different parameters. 
Acknowledgements This research was supported by the Fonds de Recherche Société et Culture and the Social Sciences and Humanities Research Council. The first author would like to thank the other authors for their invaluable help during the writing of the manuscript, as well as his family, specifically his wife and his son, who provided him with inspiration and continuous support to keep moving forward.

Open Practices Statement The data or materials for the experiment reported here will be made available, and none of the experiments was preregistered.

\section{References}

Ambadar, Z., Schooler, J. W., \& Cohn, J. F. (2005). Deciphering the Enigmatic Face: The Importance of Facial Dynamics in Interpreting Subtle Facial Expressions. Psychological Science, 16(5), 403-410. https://doi.org/10.1111/j.0956-7976.2005.01548.x

Amini, R., \& Lisetti, C. (2013). HapFACS: An Open Source API/ Software to Generate FACS-Based Expressions for ECAs Animation and for Corpus Generation. 2013 Humaine Association Conference on Affective Computing and Intelligent Interaction, 270-275. https://doi.org/10.1109/ACII.2013.51

Bassili, J. N. (1978). Facial Motion in the Perception of Faces and of Emotional Expression 4(3), 373-379.

Borod, J. C., \& Caron, H. S. (1980). Facedness and emotion related to lateral dominance, sex and expression type. Neuropsychologia, 18(2), 237-242. https://doi.org/10.1016/0028-3932(80)90070-6

Borod, J. C., Kent, J., Koff, E., Martin, C., \& Alpert, M. (1988). Facial asymmetry while posing positive and negative emotions: Support for the right hemisphere hypothesis. Neuropsychologia, 26(5), 759764. https://doi.org/10.1016/0028-3932(88)90013-9

Brockmeier, B., \& Ulrich, G. (1993). Asymmetries of Expressive Facial Movements During Experimentally-Induced Positive Vs Negative Mood States-A Video-Analytical Study. Cognition \& Emotion, 7(5), 393-405. https://doi.org/10.1080/02699939308409195

Bruce, V., \& Valentine, T. (1988). When a nod's as good as a wink: The role of dynamic information in facial recognition. In Practical aspects of memory: Current research and issues, Vol. 1: Memory in everyday life. (pp. 169-174).

Bugental, D. (1986). Unmasking the "Polite Smile": Situational and Personal Determinants of Managed Affect in Adult-Child Interaction. Personality and Social Psychology Bulletin, 12(1), 716. https://doi.org/10.1177/0146167286121001

Chamberland, C., Collin, C. (2020). An Evaluation of FaceGen Emotional Expressions: Emotional Expression Categorization of Computer-Generated and Photographic Faces with Similar Activation Unit Configurations. Manuscript submitted for publication.

Cohn, J. F., \& Schmidt, K. L. (2004). The Timing of Facial Motion in Posed and Spontaneous Smiles. International Journal of Wavelets, Multiresolution and Information Processing, 02(02), 121-132. https://doi.org/10.1142/S021969130400041X

Deese, J., \& Kaufman, R. A. (1957). Serial effects in recall of unorganized and sequentially organized verbal material. Journal of Experimental Psychology, 54(3), 180-187. https://doi.org/10.1037/ h0040536

Ekman, P. (1993). Facial expression and emotion. The American Psychologist, 48(4), 384-392. https://doi.org/10.1037/0003-066X. 48.4.384

Ekman, P. (2003). Emotions revealed: Recognizing faces and feelings to improve communication and emotional life. (2003).Emotions Revealed: Recognizing Faces and Feelings to Improve Communication and Emotional Life.Xvii, 267 Pp, 267.
Ekman, P, Friesen, W., \& O’Sullivan, M. (1988). Smiles When Lying. Paulekman. Com, 54, 414-420. https://doi.org/10.1037/0022-3514. 54.3.414

Ekman, P, Davidson, R. J., \& Friesen, W. V. (1990). The Duchenne smile: Emotional expression and brain physiology. II. Journal of Personality and Social Psychology, 58(2), 342-353. https://doi. org/10.1037/0022-3514.58.2.342

Ekman, Paul, \& Friesen, W. V. (1974). Detecting deception from the body or face. Journal of Personality and Social Psychology, 29(3), 288-298. https://doi.org/10.1037/h0036006

Ekman, Paul, \& Friesen, W. V. (1982). Felt, false, and miserable smiles. Journal of Nonverbal Behavior, 6(4), 238-252. https://doi.org/10. 1007/BF00987191

Ekman, Paul, Friesen, W. V., \& Ancoli, S. (1980). Facial Signs of Emotional Experience. Journal of Personality and Social Psychology, 39(6), 1125-1134. https://doi.org/10.1037/h0077722

Ekman, Paul, Hager, J. C., \& Friesen, W. V. (1981). The symmetry of emotional and deliberate facial actions. Psychophysiology, 18(2), 101-106.

Ekman, Paul, Friesen, W. V., \& Hager, J. C. (2002). Facial Action Coding System: The Manual. In FACS. https://doi.org/10.1016/j. msea.2004.04.064

Faul, F., Erdfelder, E., Lang, A.-G., \& Buchner, A. (2007). G*Power 3: A flexible statistical power analysis program for the social, behavioral, and biomedical sciences. Behavior Research Methods, 39(2), 175191. https://doi.org/10.3758/BF03193146

Frank, M. G., Ekman, P., \& Friesen, W. V. (1993). Behavioral Markers and Recognizability of the Smile of Enjoyment. Journal of Personality and Social Psychology, 64(1), 83-93.

Girard, J. M., Shandar, G., Liu, Z., Cohn, J. F., Yin, L., \& Morency, L.-P. (2019). Reconsidering the Duchenne Smile: Indicator of Positive Emotion or Artifact of Smile Intensity? 6.

Gosselin, P., Kirouac, G., \& Doré, F. Y. (1995). Components and Recognition of Facial Expression in the Communication of Emotion by Actors. Journal of Personality and Social Psychology, 68(1), 83-96. https://doi.org/10.1093/acprof:oso/9780195179644. 003.0012

Gosselin, P., Warren, M., \& Diotte, M. (2002). Motivation to hide emotion and children's understanding of the distinction between real and apparent emotions. The Journal of Genetic Psychology: Research and Theory on Human Development, 163(4), 479-495.

Gunnery, S. D., Hall, J. A., \& Ruben, M. A. (2013). The Deliberate Duchenne Smile: Individual Differences in Expressive Control. Journal of Nonverbal Behavior, 37(1), 29-41. https://doi.org/10. 1007/s10919-012-0139-4

Hager, J. C., \& Ekman, P. (1985). The Asymmetry of Facial Actions Is Inconsistent with Models of Hemispheric Specialization. In What the Face Reveals: Basic and Applied Studies of Spontaneous Expression Using the Facial Action Coding System (FACS) https://doi.org/10.1093/acprof:oso/9780195179644.003.0003

Hager, J. C., \& Ekman, P. (1997). The asymmetry of facial actions is inconsistent with models of hemispheric specialization. What the Face Reveals: Basic and Applied Studies of Spontaneous Expression Using the Facial Action Coding System (FACS), 40-62.

Hess, U., \& Kleck, R. E. (1990). Differentiating emotion elicited and deliberate emotional facial expressions. European Journal of Social Psychology, 20(5), 369-385.

Hess, U., \& Kleck, R. E. (1994). The cues decoders use in attempting to differentiate emotion-elicited and posed facial expressions. European Journal of Social Psychology, 24(3), 367-381. https:// doi.org/10.1002/ejsp.2420240306

Hess, U., Kappas, A., McHugo, G. J., Kleck, R. E., \& Lanzetta, J. T. (1989). An analysis of the encoding and decoding of spontaneous and posed smiles: The use of facial electromyography. Journal of Nonverbal Behavior, 13(2), 121-137. https://doi.org/10.1007/ BF00990794 
Hess, U., Blairy, S., \& Kleck, R. E. (2000). The influence of facial emotion displays, gender, and ethnicity on judgments of dominance and affiliation. Journal of Nonverbal Behavior, 24(5), 20.

Hess, U., Adams, R., \& Kleck, R. (2005). Who may frown and who should smile? Dominance, affiliation, and the display of happiness and anger. Cognition \& Emotion, 19(4), 515-536. https://doi.org/ 10.1080/02699930441000364

Kamachi, M., Bruce, V., Mukaida, S., Gyoba, J., Yoshikawa, S., \& Akamatsu, S. (2013). Dynamic properties influence the perception of facial expressions. Perception, 42(11), 1266-1278. https://doi. org $/ 10.1068 / \mathrm{p} 3131$

Krumhuber, E., \& Kappas, A. (2005). Moving smiles: The role of dynamic components for the perception of the genuineness of smiles. Journal of Nonverbal Behavior, 29(1), 3-24. https://doi.org/10. 1007/s10919-004-0887-x

Krumhuber, E., Manstead, A. S. R., \& Kappas, A. (2007). Temporal aspects of facial displays in person and expression perception: The effects of smile dynamics, head-tilt, and gender. Journal of Nonverbal Behavior, 31(1), 39-56. https://doi.org/10.1007/ s10919-006-0019-x

Krumhuber, E., Manstead, A. S. R., Cosker, D., Marshall, D., \& Rosin, P. L. (2009). Effects of Dynamic Attributes of Smiles in Human and Synthetic Faces: A Simulated Job Interview Setting. Journal of Nonverbal Behavior, 33(1), 1-15. https://doi.org/10.1007/s10919008-0056-8

Krumhuber, E. G., \& Manstead, A. S. R. (2009). Can Duchenne smiles be feigned? New evidence on felt and false smiles. Emotion (Washington, D.C.), 9(6), 807-820. https://doi.org/10.1037/ a0017844

Krumhuber, E. G., Tamarit, L., Roesch, E. B., \& Scherer, K. R. (2012). FACSGen 2.0 animation software: Generating three-dimensional FACS-valid facial expressions for emotion research. Emotion, 12(2), 351-363. https://doi.org/10.1037/a0026632

Lander, K., \& Bruce, V. (2004). Repetition priming from moving faces. Memory \& Cognition, 32(4), 640-647. https://doi.org/10.3758/ BF03195855

Mavadati, M., Sanger, P., \& Mahoor, M. H. (2016). Extended DISFA Dataset: Investigating Posed and Spontaneous Facial Expressions. 2016 IEEE Conference on Computer Vision and Pattern Recognition Workshops (CVPRW), 1452-1459. https://doi.org/10. 1109/CVPRW.2016.182

McGettigan, C., Walsh, E., Jessop, R., Agnew, Z. K., Sauter, D. A., Warren, J. E., \& Scott, S. K. (2015). Individual Differences in Laughter Perception Reveal Roles for Mentalizing and Sensorimotor Systems in the Evaluation of Emotional Authenticity. Cerebral Cortex, 25(1), 246-257. https://doi.org/10. 1093/cercor/bht227

Murdock, B. B. (1962). The serial position effect of free recall. Journal of Experimental Psychology, 64(5), 482-488. https://doi.org/10.1037/ h0045106

Niedenthal, P. M., Mermillod, M., Maringer, M., \& Hess, U. (2010). The Simulation of Smiles (SIMS) model: Embodied simulation and the meaning of facial expression. Behavioral and Brain Sciences, 33(6), 417-433. https://doi.org/10.1017/S0140525X10000865

Okamoto, H., Haraguchi, S., \& Takada, K. (2010). Laterality of asymmetry in movements of the corners of the mouth during voluntary smile. Angle Orthodontist, 80(2), 223-229. https://doi.org/10.2319/ 021809-104.1

Owren, M. J., \& Bachorowski, J. A. (2001). The evolution of emotional experience: A "selfish-gene" account of smiling and laughter in early hominids and humans. In T. J. M. G. A. Bonanno (Ed.), Emotions: Currrent issues and future directions (pp. 152-191). Guilford Press.

Roesch, E. B., Tamarit, L., Reveret, L., Grandjean, D., Sander, D., \& Scherer, K. R. (2011). FACSGen: A Tool to Synthesize Emotional
Facial Expressions Through Systematic Manipulation of Facial Action Units. Journal of Nonverbal Behavior, 35(1), 1-16. https:// doi.org/10.1007/s10919-010-0095-9

Rosenberg, E., \& Ekman, P. (1994). Coherence between expressive and experiental systems in emotions. Cognition and Emotion, 8(February), 201-230. https://doi.org/10.1080/ 02699939408408938

Ruch, W. (1995). Will the real relationsihp between facial expressoin and affective experience please stand up: The case of exhiliration. Cognition and Emotion, 9(February), 33-58. https://doi.org/10. 1080/02699939508408964

Sato, W., \& Yoshikawa, S. (2004). The dynamic aspects of emotional facial expressions. Cognition and Emotion, 18(5), 701-710. Doi https://doi.org/10.1080/02699930341000176

Sato, W., \& Yoshikawa, S. (2009). Anti-Expressions: Artificial Control Stimuli for the Visual Properties of Emotional Facial Expressions. Social Behavior \& Personality: An International Journal, 37(4), 491-501. https://doi.org/10.2224/sbp.2009.37.4.491

Schmidt, K L, \& Cohn, J. F. (2001a). Human facial expressions as adaptations: Evolutionary questions in facial expression research. American Journal of Physical Anthropology, Suppl 33, 3-24. https://doi.org/10.1002/ajpa.20001

Schmidt, K. L., \& Cohn, J. F. (2001b). Dynamics of facial expression: Normative characteristics and individual differences. Proceedings IEEE International Conference on Multimedia and Expo, 547-550. https://doi.org/10.1109/ICME.2001.1237778

Schmidt, Karen L., Cohn, J. F., \& Tian, Y. (2003). Signal characteristics of spontaneous facial expressions: Automatic movement in solitary and social smiles. Biological Psychology, 65(1), 49-66. https://doi. org/10.1016/S0301-0511(03)00098-X

Schmidt, Karen L, Liu, Y., \& Cohn, J. F. (2006a). The role of structural facial asymmetry in asymmetry of peak facial expressions. Laterality, 11 (September 2003), 540-561. https://doi.org/10.1080/ 13576500600832758

Schmidt, Karen L., Ambadar, Z., Cohn, J. F., \& Reed, L. I. (2006b). Movement differences between deliberate and spontaneous facial expressions: Zygomaticus major action in smiling. Journal of Nonverbal Behavior, 30(1), 37-52. https://doi.org/10.1007/ s10919-005-0003-x

Schmidt, Karen L., Bhattacharya, S., \& Denlinger, R. (2009). Comparison of deliberate and spontaneous facial movement in smiles and eyebrow raises. Journal of Nonverbal Behavior, 33(1), 35-45. https://doi.org/10.1007/s10919-008-0058-6

Sirota, A. D., \& Schwartz, G. E. (1982). Facial muscle patterning lateralization during elation and depression imagery. Journal of Abnormal Psychology, 91(1), 25-34. https://doi.org/10.1037/0021843X.91.1.25

Tarantili, V. V., Halazonetis, D. J., \& Spyropoulos, M. N. (2005). The spontaneous smile in dynamic motion. American Journal of Orthodontics and Dentofacial Orthopedics, 128(1), 8-15. https:// doi.org/10.1016/j.ajodo.2004.03.042

Wehrle, T., Kaiser, S., Schmidt, S., \& Scherer, K. R. (2000). Studying the dynamics of emotional expression using synthesized facial muscle movements. Journal of Personality and Social Psychology, 78(1), $105-119$.

Weiss, F., Blum, G. S., \& Gleberman, L. (1987). Anatomically based measurement of facial expressions in simulated versus hypnotically induced affect. Motivation and Emotion, 11(1), 67-81. https://doi. org/10.1007/BF00992214

Publisher's note Springer Nature remains neutral with regard to jurisdictional claims in published maps and institutional affiliations. 